Results: 46 patients satisfied criteria for $A D$ ( $34 \mathrm{~F}$ and $12 \mathrm{M}$ ), 57 for $\mathrm{VaD}$ $(29 \mathrm{~F}$ and $28 \mathrm{M})$ and 26 patients had CDND $(12 \mathrm{~F}$ and $14 \mathrm{M})$. $55.8 \%$ of subjects had hypertension (14 CDND, SBP $142 \pm 17 \mathrm{mmHg}$, DBP $81 \pm 7 \mathrm{mmHg}$; $17 \mathrm{AD}$, SBP $135 \pm 21 \mathrm{mmHg}$, DBP $79 \pm 7 \mathrm{mmHg}$ and $36 \mathrm{VaD}, \mathrm{SBP} 140 \pm 18 \mathrm{mmHg}$, DBP $81 \pm 7 \mathrm{mmHg} ; \mathrm{P}=\mathrm{n} . \mathrm{s}$.). $\mathrm{HDL}$-cholesterol levels were significantly different in the three groups (CDND $43.44 \pm 11.39 \mathrm{mg} / \mathrm{dl}$, AD $51.71 \pm 14.31 \mathrm{mg} / \mathrm{dl}$, VaD $43.64 \pm 14.58 \mathrm{mg} / \mathrm{dl} ; \mathrm{P}<0.05$ ), while no differences in total cholesterol, LDL cholesterol and triglycerides were detected. AD patients showed significantly lower BDNF levels compared to CDND subjects (CDND $363.5 \pm 143.4 \mathrm{pg} / \mathrm{ml}$, AD $264.6 \pm 147.6 \mathrm{pg} / \mathrm{ml}, \mathrm{VaD} 304.1 \pm 121.8 \mathrm{pg} / \mathrm{ml} ; \mathrm{P}<0.02$ ), also after adjustment for age and sex. DM had significantly lower BDNF levels compared to NDM (DM 271.4 $\pm 129.8 \mathrm{pg} / \mathrm{ml}$, NDM $313.9 \pm 142.3 \mathrm{pg} / \mathrm{ml} ; \mathrm{P}=0.12$ ). Among NDM subjects, patients with dementia ( $A D$ and $\mathrm{VaD}$ ) had significantly lower BDNF levels compared to CDND patients (CDND $398.7 \pm 148.2 \mathrm{pg} / \mathrm{ml}$, AD $290.5 \pm 138.5 \mathrm{pg} / \mathrm{ml} ; \mathrm{P}<0.03, \mathrm{VaD} 297.8 \pm 132.4 \mathrm{pg} / \mathrm{ml} ; \mathrm{P}<0.03)$. Among $D M$ patients, those patients with $A D$ showed significantly lower BDNF levels compared to VaD and CDND subjects (CDND 293.3 $\pm 109.6 \mathrm{pg} / \mathrm{ml}, A D$ $176.8+150.7 \mathrm{pg} / \mathrm{ml}, \mathrm{VaD} 316.7+98.9 \mathrm{pg} / \mathrm{ml} ; \mathrm{P}<0.02)$.

Conclusions: Reduced circulating levels of BDNF in diabetic patients and in subjects with primary dementia could suggest that reduced levels of neurotrophin could link T2D to cognitive disorders, potentially explaining the clustering of these conditions in epidemiological studies.

\section{ESTIMATION OF CARDIOVASCULAR RISK IN TYPE 2 DIABETES}

E. Pellegrini, M.S. Simonini, M. Maurantonio, I.M. Giannico, R. D’Amico, D. Ganazzi, L. Carulli, P. Loria, M. Bertolotti, N. Carulli. Università di Modena e Reggio Emilia, Modena, Italy

E-mail: pelleg@tsc4.com

Diabetes mellitus is a major risk factor for cardiovascular (CV) events. Many algorithms have been devised to assess CV risk, some of which specific for diabetics. Most of them, however, can hardly be extrapolated to Mediterranean countries.

Aim of this study was to analyze CV risk and the incidence of CV events in a local cohort of patients with type 2 diabetes.

Methods: Clinical charts of the Diabetes Clinics of Modena in the period 1991-1995 were analyzed. Patients aged 35-65 with type 2 diabetes and no previous CV disease were eligible. Global CV risk was computed according to Framingham, RISCARD, Progetto Cuore and UKPDS algorithms and compared with the actual rate of $\mathrm{CV}$ events over the following 10 years.

Results: 2416 patients were screened; 1532 of them (63.4\%) were eligible on the basis of predefined criteria and completeness of data. In such population an absolute 10 -yr risk rate of $14.6 \%$ was observed. When looking at the characteristics of the patients who developed a cardiovascular event compared to those who did not, we found a significant difference in the prevalence of risk factors as systolic blood pressure, age at visit, smoke, duration of diabetic disease and HbA1c. COPD and chronic heart failure also display a higher prevalence in patients with events, suggesting a possible role of these chronic conditions in developing cardiovascular disease. Interestingly, most of the subjects presenting with a CV event had a low to moderate risk estimate at the beginning; this was particularly evident with the Progetto Cuore algorithm.

Conclusions: Estimation of $\mathrm{CV}$ risk is largely dependent on the algorithm adopted and on the baseline risk of the reference cohort. Equations designed for a specific population should be adopted. The overall performance of presently available functions is however low. Inclusion of additional risk parameters might hopefully increase the performance of such algorithms, which is presently clearly unsatisfactory. The algorithm derived from the present study will be utilized for a prospective evaluation of CV risk in our local cohort.

\section{APOE MODENA: A NOVEL APOE MUTANT CAUSING LIPOPROTEIN GLOMERULOPATHY}

E. Pellegrini ${ }^{1}$, M. Bertolotti ${ }^{1}$, R. Magistroni ${ }^{1}$, J. Piattoni ${ }^{1}$, L. Carulli ${ }^{1}$, G. Russi ${ }^{2}$, L. Pisciotta ${ }^{3}$, S. Calandra ${ }^{1}$, S. Bertolini ${ }^{3} .{ }^{1}$ University of Modena \& Reggio Emilia; ${ }^{2}$ City Hospital, Reggio Emilia; ${ }^{3}$ University of Genoa, Italy E-mail: bertolotti.marco@unimore.it

Lipoprotein glomerulopathy (LPG) is a rare disease characterized by laminated lipid thrombi in the lumina of dilated glomerular capillaries. Apolipoprotein $E$ and $B$ can be demonstrated in these lipid deposits. The plasma lipid profile of LPG patients is similar to that of dysbetalipoproteinemia with elevated IDL and ApoE. Patients often present nephrotic proteinuria but their lipid profile differs from that of nephrotic syndrome secondary to other kidney diseases. LPG has been mainly reported in Japanese and Chinese subjects, associated with novel mutations in APOE gene encoding ApoE.

We have identified LPG in an Italian woman who at the age of 47 was found to have a combined hyperlipidemia (TC 376 and TG $306 \mathrm{mg} / \mathrm{dl}$ ) and subsequently developed proteinuria in the nephrotic range. Renal biopsy demonstrated lipoprotein thrombi in glomerular capillaries suggesting LPG. The sequence of APOE gene showed that the patient was: (i) homozygous for epsilon-2 allele [Cys112 and Cys158 in the mature protein] and (ii) heterozygous for a novel mutation in exon 4: c.502 C>T [Arg 150>Cys in the mature protein]. Since the mutant ApoE has a new cysteine residue it is likely that it forms a disulfide bridge with the other Cys residues of the E2 isoforms, resulting in
ApoE polymerization (dominant negative effect). This is probably the cause of both dyslipidemia and lipid thrombi in the glomerular capillaries. In view of the poor response of plasma lipids and renal histology and function to statin treatment, the patient started lipid-apheresis with reduction of both dyslipidemia and proteinuria.

\section{CIRCULATING OSTEOGENIC CELLS AS A MAJOR DETERMINANT OF} ARTERIAL STIFFENING IN POST-MENOPAUSAL OSTEOPOROSIS

M. Pirro, M.R. Mannarino, R. Helou, G. Fabbriciani, C. Leli, L. Callarelli, M.R. Manfredelli, A.M. Scarponi, E. Mannarino. Internal Medicine, Angiology and Arteriosclerosis Diseases, University of Perugia, Italy

E-mail: mpirro@unipg.it

Aim: The maintenance of stable bone mass during adult life is dependent from the activity of bone resident osteogenic cells, the osteoblasts (OBs), which are able to mineralize in vitro and form bone in vivo. Impaired osteoblast recruitment and function have been observed in subjects with osteoporosis, which have also stiffer arteries than non osteoporotic subjects. Osteoblast lineage cells with stem cell properties express alkaline-phosphatase and CD34 surface antigens; they have been recently identified in the peripheral blood of normal subjects, thus circulating in direct contact with the inner arterial surface. Whether circulating OBs may contribute to arterial stiffening in osteoporotic post-menopausal women is unknown.

Methods: The number of circulating OBs was quantified in peripheral blood by FACS analysis in 50 post-menopausal osteoporotic women and 35 ageand sex-matched non-osteoporotic controls. OBs were defined as double positive alkaline-phosphatase and CD34 cells. All participants underwent cardiovascular risk assessment, measurement of femoral neck and total hip bone mineral density (BMD) by Dual-Energy X-ray Absorbptiometry, aortic pulse wave velocity (aPWV) as an indirect index of arterial stiffness, and plasma PTH levels.

Results: Levels of circulating double positive alkaline-phosphatase and CD34 $\mathrm{OBs}$ and aPWV were higher in osteoporotic post-menopausal women than in non-osteoporotic controls $(2.72 \pm 0.77 \mathrm{n} / \mathrm{microL}$ vs $0.72 \pm 0.12 \mathrm{n} / \mathrm{microL}$, $\mathrm{p}=0.04 ; 10.4 \pm 0.45 \mathrm{~m} / \mathrm{s}$ vs $9.1 \pm 0.32 \mathrm{~m} / \mathrm{s}, \mathrm{p}=0.03$ ), whereas PTH levels were comparable in the two groups. In the whole cohort, the number of circulating OBs was positively correlated with aPWV ( $r$ ho $=0.35, p=0.002)$ and negatively with femoral neck BMD $(r=-0.27, p=0.01)$ and total hip BMD $(r=-0.30$, $p=0.007$ ). Femoral neck $B M D$ and total hip $B M D$ were inversely correlated with aPWV $(r=-0.34, p=0.003$ and $r=-0.32, p=0.005$, respectively), whereas aPWV and PTH levels were positively correlated $(r h o=0.31, p=0.007)$. Linear regression analysis showed that an increased number of double positive alkaline-phosphatase and CD34 OBs, age and systolic blood pressure were all significant independent predictor of aPWV (Model $\mathrm{R}=0.56, \mathrm{p}<0.001$ ), independent of PTH levels.

Conclusions: In women with post-menopausal osteoporosis an increased availability of circulating osteoblasts may reflect the possible influence of bone-marrow stem cells to bone formation. However, the presence of increased levels of immature osteoblasts in the blood stream may have a detrimental impact on arterial compliance, possibly as a consequence of circulating osteoblast contribution to arterial wall calcification.

\section{NEW LDLR GENE MUTATIONS CAUSING FH IN ITALY}

L. Pisciotta ${ }^{1}$, A. Bellocchio ${ }^{1}$, R. Fresa ${ }^{1}$, C. Rabacchi ${ }^{2}$, A. Wunsch ${ }^{2}$, I. Blotta ${ }^{3}$, A. Cantafora ${ }^{3}$, S. Calandra' ${ }^{2}$, S. Bertolini ${ }^{1} .{ }^{1}$ Dpt. of Internal Med., Univ. of Genoa; ${ }^{2}$ Dpt. of Biomed. Sci., Univ. of Modena and R. Emilia; ${ }^{3}$ Nat. Inst. of Health, Rome, Italy

E-mail: livia.pisciotta@unige.it

$\mathrm{FH}$ is mainly due to mutations in LDLR gene. Diagnosis is based on evaluation of family history, patient's clinical history, physical examination and high level of LDL-C. We collected 100 new probands with probable diagnosis of FH that were screened by DHPLC for mutations causing FDB and by "Surveyor method" or direct sequencing for mutations in LDLR gene. In absence of minute or point mutations in LDLR gene, we used MLPA to detect major rearrangements. We identified the following LDLR novel mutations never described before: A $>$ C -215 from ATG; c. 130-131 ins GT>Fs21 >162aa >X185; c. 185-186 ins T>Fs41>66aa>X108; c.296-297 del CA>S78X; c.382 G>T, C100F; c.366-367 ins C>Fs102>X108; C.391 G>C, D110H; c.431-434 dupl CGGT>Fs125>X159; c.616-617 ins. A>Fs185>X196; C.648 ins T, D196X; c.676-684 dupl, dupl S205, D206, E207; IVS4 c.694 +3 ins T; c. 968 del G>Fs301>X347; C. 1130 G>C, C356S IVS8 c.1187 -1 G>A; c.1211 C>T, T383I; c.1230 G>C, R389S; c.1296-1298 de GGA, del D412; c.1570 G>A, V503M; c.1586 G>A, G508D; c.1644 T>A, N527L; c.1721 G>A, R553H; c.1859 G>A, W599X; c.1978 C>A, Q639L; c.2171 C>T, T703l; c. 2225 del C>Fs721>X743; IVS16 c. $2389+5$ G>A; IVS16 C. 2390 -1 G>A. We also identified for the first time in Italy the following mutation previously described elsewhere: exons 5-6 del; c. 58 G>A, G-2>R; IVS1 c.68 -1 G>C; c.661 G>T, D200Y; c.681 C>G, D206E; c. 953 G>A, C297T; c. 977 C >G, S305C; c.1048 C>T, R329X; c.1104 C>A, C347X; c.1246 C>T, R395W; c.1257 C>G, Y398X; c.1285 G>A, V408M; c.1474 G>A, D471N; c.1618 G>A, A519T; c.1879 G>A, A606T; IVS13 c.1988 -2 A>G; c.2132 G>A, C690T; c.2389 G>A, V776M. 